\title{
Leaders
}

\section{Papillomavirus research update: highlights of the Barcelona HPV 2000 international papillomavirus conference}

F X Bosch, T Rohan, A Schneider, I Frazer, H Pfister, X Castellsagué, S de Sanjosé, V Moreno, L M Puig-Tintore, P G Smith, N Muñoz, H zur Hausen

\begin{abstract}
Servei
d'Epidemiologia i

Registre del Càncer, Institut Català

d'Oncologia, Av Gran

Via KM 2,7, E.08907

L'Hospitalet de

Llobregat, Barcelona,

Spain

F X Bosch

X Castellsagué

$\mathrm{S}$ de Sanjosé

V Moreno
\end{abstract}

Department of

Epidemiology and

Social Medicine,

Albert Einstein

College of Medicine,

New York NY-10461,

USA

T Rohan

Department of

Gynaecology,

Friedrich Schiller

University D-07743,

Jena, Germany

A Schneider

Centre for

Immunology and

Cancer Research,

University of

Queensland, 4102

Brisbane, Australia

I Frazer

Institute of Virology, University of Koln, D-50935 Koln,

Germany

$\mathrm{H}$ Pfister

Secció de Ginecologia Oncològica ICGON, Hospital Clínic

Universitari, E-08036

Barcelona, Spain

L M Puig-Tintore

continued over

Correspondence to: Dr Bosch

x.bosch@ico.scs.es

Accepted for publication 6 December 2000

\begin{abstract}
The 18th international papillomavirus conference took place in Barcelona, Spain in July 2000. The HPV clinical workshop was jointly organised with the annual meeting of the Spanish Association of Cervical Pathology and Colposcopy. The conference included 615 abstracts describing ongoing research in epidemiology, diagnosis/screening, treatment/prognosis, immunology/human immunodeficiency virus, vaccine development/trials, transformation/progression, replication, transcription/translation, viral protein functions, and viral and host interactions. This leader summarises the highlights presented at the conference (the full text of the abstracts and lectures can be found at www.hpv2000.com). Relevant material in Spanish can be found at www.aepcc. org.

(F Clin Pathol 2001;54:163-175)
\end{abstract}

Keywords: papillomavirus; epidemiology; immunology; biology; screening

The 18th international papillomavirus conference took place in Barcelona, Spain in July 2000. The human papillomavirus (HPV) clinical workshop was jointly organised with the annual meeting of the Spanish Association of Cervical Pathology and Colposcopy. The conference included 615 abstracts describing ongoing research as follows: epidemiology (127 abstracts), diagnosis/screening (129 abstracts), treatment/prognosis (39 abstracts), immunology/human immunodeficiency virus (HIV) (61 abstracts), vaccine development/ trials (56 abstracts), transformation/progression (53 abstacts), replication (20 abstracts), transcription/translation (30 abstracts), viral protein functions (40 abstracts), and viral and host interactions (60 abstracts). A special workshop devoted to presenting ongoing research in developing countries was also organised and included 138 abstracts. The conference also featured 80 invited lectures. The highlights presented at the conference were selected and are briefly reported below. The full text of the abstracts and lectures can be found at www.hpv2000.com and the numbers in parenthesis in the text below refer to the abstract number on the website. Key publications for further reading are listed at the end of this paper. ${ }^{1-19}$

The opening session was given by PG Smith (London School of Hygiene and Tropical Medicine) who lectured on vaccination trials in developing countries. Professor Smith stressed that the next decade holds the promise of new vaccines against some of the major infectious diseases affecting mankind. Most of the research and deployment efforts are, and will continue to be, directed at vaccines against the major infectious diseases, such as malaria, tuberculosis, pneumonia, meningitis, and AIDS. However, some will be directed at what have traditionally been called "chronic" diseases, an increasing number of which appear to have an infectious aetiology. Hepatitis B vaccination should have a profound influence on the rates of chronic liver disease and liver cancer. Similarly, the aetiological association between HPV and cervical cancer has become increasingly clear and vaccines that prevent or treat these infections are on the near horizon.

The need for HPV vaccines is greatest in areas of high incidence of cervical cancer and especially in those settings where diagnostic and treatment services are poor. In most of the industrialised countries effective screening and treatment programmes for early lesions have the potential to reduce cervical cancer mortality rates to low levels, but even in these countries it is often the case that those at highest risk of disease are least likely to enter screening programmes. In the developing countries, the resources for screening programmes are sparse, as is access to effective treatment for any lesions identified. An efficacious vaccine against HPV infections, especially one that acted against established infections, as well as preventing new ones, could have a rapid and profound impact on one of the major cancers afflicting women globally.

Before new vaccines are introduced into widespread use it is essential that their safety and efficacy should be properly established. This will require large randomised controlled trials, usually double blind, preceded by 
Department of Infectious and Tropical Diseases, London

School of Hygiene and

Tropical Medicine,

London WC1E 7HT,

UK

P G Smith

Unit of Field and Intervention Studies, International Agency for Research on Cancer, F-69372 Lyon, France N Muñoz

Deutsche Krebsforschungszentrum, D-69120 Heidelberg, Germany $\mathrm{H}$ zur Hausen appropriate smaller scale studies (preclinical and phase I/II trials) to establish immunogenicity and to detect any major adverse effects. Initial trials may be conducted in developed countries, where the candidate vaccines are most likely to be produced, at least initially. But even if they are found to be safe and efficacious in these settings it cannot be assumed that the same efficacy will hold in other geographical and ecological situations. Further large scale trials will be required in developing countries, where the main market for the vaccines is likely to be, in public health terms if not economically.

Special methods need to be developed to ensure that individuals included in trials, many of whom may be illiterate, can be followed up and the endpoints of interest ascertained, often many years after an individual has entered into the trial. Furthermore, in such situations, major ethical issues need to be dealt with. The level of medical care that must be provided for those in a trial has been the subject of heated debate.

Conducting trials of new "expensive" vaccines in developing countries has been considered unethical because it is unlikely that those countries will be able to afford to include a vaccine that is shown to be efficacious in the routine vaccination programme. An issue that must be discussed before a trial starts is the extent to which an efficacious vaccine will be made available after a trial. At the very least those in the "control" group should be offered the vaccine, if they are still likely to benefit. However, many consider the responsibility of the sponsors goes beyond this to the wider community. It is common now to incorporate a cost-benefit analysis into field trials of new interventions.

Most existing vaccines that are in widespread use in developing countries are directed at infections usually acquired in infancy or childhood. However, it seems unlikely that HPV vaccines will be directed at this age group, at least initially. The target group, for preventive vaccines, is likely to comprise girls or young women shortly before the onset of sexual activity. This age group is more difficult to recruit into trials, especially because in many developing countries girls of this age are unlikely to be in school. Those planning HIV vaccine trials will have similar difficulties and the groundwork already done in preparing for HIV trials may be of great value for the planning of HPV vaccine trials. Indeed, a successful HPV vaccine looks to be closer than an HIV vaccine.

\section{Epidemiology}

The results of combined analyses of the International Agency for Research on Cancer (IARC) based multicentric case control study were presented (Muñoz et al, 053). The studies included 2288 cases and 2513 controls from seven countries and used advanced polymerase chain reaction (PCR) technology for HPV detection and typing. The combined odds ratio (OR) for viral detection was $83.3 \quad(95 \%$ confidence interval (CI) 54.9 to 105.3 ) and the ORs for the most relevant viral types were:
HPV-16, 182; HPV-18, 231; HPV-45, 148; and HPV-31, 71.5. The study updated the conclusions of the HPV IARC monograph providing the strongest evidence to support the identification of HPV types 16, 18, 31, 33, 35, 45, 51, 52,58 , and 59 as human carcinogens.

Most of the epidemiology presentations were on genital HPV infections and cervical neoplasia. One descriptive epidemiological report of interest was from Lazcano-Ponce et al (100) who, in studying a population based sample of 1340 Mexican women, observed two peaks of HPV DNA prevalence, one in women aged $<25$ years, and the other in women over the age of 65 years, with the lowest prevalence being seen in women aged 35-44 years. In all age groups, high risk HPV types predominated. These data are intriguing because they seem to contradict the previously widely accepted notion; namely, that HPV prevalence declines with age. They are, however, in agreement with data from a recent JNCI report expanded by an abstract by Herrero et al (054) from the Costa Rica study, and with those from some of the studies included in his international prevalence survey reported at this meeting. The interpretation of the peak in prevalence in older women is not clear, but could represent either a cohort effect or reactivation of latent infection. Evidence for this being a cohort effect was provided by Peto et al (308), who showed a positive correlation between the HPV prevalence by birth cohort and the corresponding lifetime risk of dying from cervical cancer in the UK.

In work that has some bearing on the Bethesda system, Peto et al (179) presented data from the Manchester cohort showing that the prevalence of cervical intraepithelial neoplasia 3 (CIN3) increased linearly with increasing time since last normal smear (for intervals up to six years), suggesting therefore that the true incidence of new disease can be estimated reliably in a screened cohort and that CIN3 cases persist for at least this interval. The prevalence of other abnormalities, including notably CIN2, was independent of the screening interval and essentially unrelated to HPV in a previous normal smear, suggesting that these abnormalities are part of the normal morphological spectrum of transient HPV infection. If correct, this would suggest that the inclusion of CIN2 in the high grade squamous intraepithelial lesion (HSIL) category of the Bethesda classification system might be both a clinical and scientific error, a conclusion supported by some recent cohort data from Toronto.

HPV serology raised some attention at the meeting. Two population based studies by Herrero et al, one in Argentina (165) and one in Thailand (167), examined correlates of type specific virus-like particles (VLPs). Both studies were similar in design and both showed that the prevalence of antibodies against VLPs of HPV types 16, 18, 31, 33, and 58 increased with increasing number of sexual partners. They also showed a strong correlation between the detection of antibodies against a specific HPV type and the presence of the same type in 
the cervix, and that there is considerable crossreactivity. Antibodies were rare in virgins. In a study of anti-VLP-16 responses in young women, Ho et al (058) showed that those with persistent IgG antibodies to HPV-16 VLP were half as likely to acquire HPV of any type, suggesting that persistent antibody may cross protect against infection with other HPV types. Thomann et al (157), in the Ludwig-McGill cohort, showed that $\operatorname{IgM}$ seropositivity at enrolment was predictive of the risk of acquisition of incident HPV-16 infection, whereas IgG seropositivity was more consistently associated with prevalent or persistent oncogenic HPV infection. IgM seropositivity had no diagnostic value with respect to the detection of HPV infection or cervical lesions.

In an intriguing report, Lewis et al (145) described the use of capture enzyme linked immunosorbent assay (ELISA) to study antibodies against HPV types 6/11 and 16 in saliva, oral mucosal transudates, and serum. IgG antibodies to HPV types $6 / 11$ and 16 were detected less frequently in the oral samples than in serum, possibly because of suboptimal collection of oral specimens or because antibody concentrations in the oral specimens are truly lower. Nevertheless, HPV IgG values in oral specimens correlated with seropositivity, and if this approach can be optimised, it is conceivable that oral samples could be useful in epidemiological studies as a non-invasive alternative to serological specimens.

Some data were presented on infections with multiple HPV types, which is of interest in relation to vaccine development. For example, in the Ludwig-McGill cohort, Rousseau et al (137) showed that multiple infections were relatively common, occurring in about $19 \%$ of women each visit. Furthermore, assuming independence of types, she observed an excess of joint cumulative positivity of types involving HPV types 16, 31, and 53, suggesting that individual type infections are not independent. To a large extent, risk factors for single and multiple infections were similar, although age and number of sexual partners were more strongly associated with multiple infections than with single infections. In addition, Slavinsky et al (146) showed that HIV positive women were more likely to be infected with multiple HPV types than HIV negative women.

Bosch et al (064) presented data from the IARC multicentre case control study of HPV and cervical adenocarcinoma, the largest of such studies to date. He showed a very strong positive association between HPV infection and risk (OR, $68.7 ; 95 \% \mathrm{CI}, 36.2$ to 130.5$)$ and similarly high ORs for individual HPV types. The report also showed that risk factors for adenocarcinoma are largely the same as those for squamous cell carcinoma, the exception being an apparent inverse association between parity and risk. An observation of interest, consistent with the results of squamous cell carcinomas, is the likely interaction between long term use of oral contraceptives (OCs) and cervical adenocarcinoma.
COFACTORS IN CERVICAL CARCINOGENESIS Cofactors continue to be of interest in relation to HPV related cervical neoplasia because not all HPV positive women develop cervical cancer. In this regard, Moreno et al (061), using data from the IARC multicentric case control study, showed strong evidence for the role of OCs as a late stage factor in HPV induced carcinogenesis. According to this study, women who had used OCs for six or more years and were found to be positive for a high risk HPV type had a four times greater risk of developing squamous cell cervical cancer than women with HPV DNA who were not exposed to OCs. OCs did not modify the chances of being HPV positive among controls. This finding might have clinical implications. Two studies suggested that Chlamydia trachomatis infection might increase the risk of invasive squamous cervical cancer. Wallin et al (113) presented results from a prospective study in Sweden in which $C$ trachomatis detected in cervical smears collected on average 5.6 years before diagnosis or termination of follow up was associated with a 17-fold increase in risk of invasive cervical cancer after adjustment for HPV status, and Smith et al (071) found a positive association between $C$ trachomatis seropositivity and invasive squamous cervical cancer risk in HPV positive subjects in case control studies in Brazil and the Philippines, with the ORs increasing with increasing $C$ trachomatis antibody titres. In contrast, van den Brule et al (063) found no association between genital $C$ trachomatis infection and risk of subsequent HSIL in HPV positive women, but a possible association with the risk of abnormal squamous cells of indetermined significance/low grade squamous intraepithelial lesion (ASCUS/LSIL), and Moscicki et al (075) did not show an association between $C$ trachomatis (and other sexually transmitted diseases) and the risk of HSIL. If, indeed, $C$ trachomatis does influence the development of cervical neoplasia, the mechanism by which it does so is not clear, but might involve the induction of a chronic inflammatory process. In relation to this, Castle et al (070) presented evidence from the Costa Rican cohort suggesting that cervical inflammation was positively associated with the risk of the progression of oncogenic HPV to HSIL or carcinoma. They also found that bacterial vaginosis was inversely associated with the risk of progression. In terms of other possible cofactors, Smith et al (082) also found a positive association between herpes simplex virus 2 (HSV-2) serology and cervical cancer risk overall, and in HPV positive subjects in the Philippine component but not the Brazilian component of their Brazilo-Philippine study.

One phenomenon of interest in relation to risk of cervical cancer is the so called "male" factor. Essentially, this is taken to mean that the sexual behaviour of men (for example, the number of sexual partners that they have had) can influence the risk of cervical cancer in their female sexual partners. In this regard, Bleeker et al (140) presented data showing that $80 \%$ of the male sexual partners of women with CIN had penile lesions, of which a substantial 
proportion were infected with HPV, and they speculated that penile lesions in sexual partners of women with CIN are probably productive and that they might play an important role in influencing the course of cervical lesions in these women by continuously reinfecting them with HPV. Castellsagué et al (060), in a pooled analysis of data from the seven IARC multicentre case control studies of CIN3 and invasive cancer, showed that the prevalence of penile HPV was generally higher in husbands of cases than in husbands of controls. However, penile HPV was not associated with the risk of cervical neoplasia in high or intermediate risk countries, but was associated with an eightfold increase in risk in Spain.

HPV AND SKIN CANCER

During the past few years, the association of HPV with skin cancer in the general population apart from the epidermodysplasia verruciformis (EV) syndrome has been a subject of intense research. Non-melanoma skin cancer is the most frequent cancer among white individuals worldwide and is particularly widespread among the increasing number of immunosuppressed patients. Using newly developed, highly sensitive PCRs, a large and still growing number of new partial sequences could be identified in non-melanoma skin cancers, which all probably represent new PV types. Most of them are related to EV associated viruses but, depending on the PCR used, HPV-4 related types have also been identified (Antonsson et al, 116). These days, genital HPV types are less frequently detected. The HPV prevalence is highest in squamous cell carcinomas of immunosuppressed patients (up to $90 \%$ ) and lowest in basal cell carcinomas of immunocompetent patients (about 40\%, which is close to the prevalence in normal skin samples) (Rust et al, 497; Wieland et al, 115). Viral load determinations revealed only one HPV DNA copy/20-1000 cells in the tumour (Wieland et al, 115; Stockfleth et al, 125), indicating that probably not every tumour cell harbours an HPV genome. This suggests that the contributions of cutaneous HPV types to carcinogenesis are quite different from genital $\mathrm{PVs}$ in cervical cancer. It underlines the need for studies of the molecular biology of these viruses so that their activity can be better understood. Preliminary experiments to this end were presented that dealt with the biochemical functions of cutaneous HPV proteins, with the ultraviolet light effect on viral promoter activity, and with the effects of cutaneous HPV oncoproteins in organotypic cultures (Caldeira et al, 604; Ruhland and de Villiers, 661; Mulder et al, 629, respectively).

NATURAL HISTORY OF HPV INFECTION IN THE SKIN

The frequent detection of viral DNA identical with or related to EV associated HPVs in healthy skin samples or plucked hairs of individuals in all age groups supports the notion that probably everybody is infected over long periods, if not throughout life, with these viruses. The general need for highly sensitive detection methods (usually nested PCR) indicates that these lingering viruses replicate at very low levels, which helps them to escape from immune detection. However, the detection of antibodies to capsid L1 protein indicates that productive infection does takes place (Feltkamp et al, 102). Persistent infections are obviously activated by sunlight exposure, immunosuppression, or hyperproliferation of the epithelium such as in psoriasis. An interesting report on the transient generation of anti-HPV-5 antibodies after epidermal repair in burns supports the view of transient activation of persisting viruses as a result of epidermal proliferation (Favre et al, 676). A similar scenario has been proposed for the cervical-vaginal mucosa by Jin et al (197), who found a strikingly high prevalence of HPV types 61,72 , and 11 related, novel sequences in lavages of HIV infected women. These previously rare members of the phylogenetic subgroup A3 seem to be activated during AIDS associated immunodeficiency, but must certainly have a reservoir in the general population. The growing awareness of this type of ubiquitous infection will have a strong impact on future plans for disease prevention and treatment.

HPV AND CANCERS OF THE ORAL CAVITY AND THE ANAL AND PERIANAL REGIONS

In relation to epidemiological presentations on HPV and cancer sites other than the cervix, the study by Björge et al (148) provided the first prospective evidence that previous infection by HPV-16 (as indicated by the presence of IgG antibodies in serum samples collected on average 10 years before cancer diagnosis) is associated with an increased risk of anal or perianal cancers.

There were several presentations on various aspects of HPV and oropharyngeal cancers. An early report by Herrero et al (166) from the IARC international case control study of HPV and cancer of the oral cavity and pharynx suggested that HPV is not a major aetiological factor, based on the comparison of cases and controls with respect to HPV prevalence in exfoliated cells. However, the HPV detection rate was three times higher in tumour biopsies than in exfoliated cells. In contrast, Mork et al (065), in a case control study nested within a Nordic cohort of almost 900000 subjects who had donated serum samples, showed that HPV-16 positivity in serum collected on average 9.4 years before diagnosis was associated with a 14-fold increase in the risk of subsequent oropharyngeal cancer (OR, 14.4; 95\% CI, 3.6 to 58.1 ). Seropositivity to HPV types 18,33 , and 73 was not associated with altered risk. The reason for the discrepancy between the findings of these two studies is unclearpossible explanations include differences in study design or specimen types, differences in the methods of assessing HPV status, or lack of good information on confounders in the Nordic study. The preliminary results of the IARC study are consistent with previous studies indicating that HPV might have aetiological 
relevance for tonsillar carcinomas. This is supported to some extent by the results of HPV analysis of tumours in the case series reported by Weissenborn et al (114) and Summersgill et al (172), who found HPV DNA in $58 \%$ and $63 \%$, respectively, of tonsillar tumours.

CERVICAL CANCER IN DEVELOPING COUNTRIES The 18th international conference included for the first time a special workshop entitled "HPV in developing countries: screening, diagnosis, therapy and prevention". The workshop was chaired by $\mathrm{T}$ Wright (University of Columbia) and R Sankaranarayanan (IARC) and included 138 abstracts.

The presentations in this workshop dealt mainly with the prevalence and type distribution of genital HPV infections in central America and in Africa, and in the quality of cytological screening for cervical neoplasia in developing countries. The former did not allow firm conclusions to be drawn concerning differences in HPV type distribution from those reported elsewhere, and the latter highlighted the need for improvements in the quality of screening, which is handicapped by the lack of resources in developing countries. Clearly, these are important topics given the relatively high rates of cervical cancer in these areas, and most presentations highlighted the need for further investigations in these areas. Visual inspection of the cervix was discussed extensively at the meeting. Ferreccio et al (200) described a cervical cancer prevention programme in Peru that uses visual inspection of the cervix with acetic acid and immediate treatment - it is anticipated that this will lead to a far higher detection and treatment rate for high grade lesions than in neighbouring control areas in which Papanicolaou (Pap) based programmes operate. It is expected that further developments in this important area of field research and implementation will be stimulated by the growing presence of international cooperative networks such as the Alliance for Cervical Cancer Prevention (PAHO, PATH, IARC, AVSC Int. JHPIEGO (www.alliancecxca.org)), the Challenge/ESO Group (www. cancerworld.org), and the International Network for Control of Gynaecological Cancers (www.matweb.hcuge.ch/matweb).

\section{Diagnosis and detection of HPV DNA}

Several papers concerned new PCR based techniques for HPV detection. The accurate detection of various HPV types was described by van Doorn et al (254 and 255), where a broad spectrum PCR primer set (SPF10) is used to amplify a fragment of $65 \mathrm{bp}$ from the L1 region. Genotyping is done by reverse hybridisation on a line probe assay discriminating between $25 \mathrm{HPV}$ genotypes. Seth et al (253) described the application of the same technique to 12 year old archived smears; $31 \%$ of cases were HPV positive, with higher rates in women born after 1940 compared with those born before 1940 . The SPF10 system was more sensitive than the GP5+/6+ system. To facilitate the detection of a wide range of various HPV types, 11 blocks of conserved L1 protein sequences were identified (Baines et al, 126). Specific primers were designed which, in contrast to the MY 09/11 and the GP5+/6+ system, allowed the detection of PVs in all groups of the HPV Sequence Database. To improve sensitivity, a PCR system was designed by Kornegay et al (282), where a non-degenerate pool of five upstream and seven downstream primers was designed in conserved regions of the $\mathrm{L} 1$ gene, resulting in a $170 \mathrm{bp}$ product. In clinical specimens, the short amplification detected up to $10 \%$ more HPV positives compared with the MY 09/11 system.

LOCALISATION OF HPV DNA WITHIN TISSUE SPECIMENS AND VIRUS ASSOCIATED PROGRESSION MARKERS

A combination of the SPF10 system with in situ hybridisation (Vermeulen et al, 236) allowed the detection and localisation of multiple HPV infections in up to $20 \%$ of HPV positive cervical cancers. In situ hybridisation results indicated the presence of multiple HPV subclones.

Several studies looked at the potential value of virus associated progression markers, such as the presence of certain viral RNA species or the E4 protein (Sotlar et al, 195), viral load (Swan et al, 280), and integration (Klaes et al, 194; Counio Coste et al, 223; Blennow et al, 231). Lamarcq et al (187) showed that the expression of E6/E7 transcripts and vascular endothelial growth factor (VEGF) mRNA was higher in women with dysplastic cervical smears than in those with normal smears, suggesting that these markers might be a useful prognostic and diagnostic tool for cervical disease. Because for most of these markers only data from cross sectional studies are available at this point, their clinical usefulness is still unknown.

NEW BIOMARKERS AS A QUALITY CONTROL OF THE HISTOLOGICAL OR CYTOLOGICAL DIAGNOSIS A non-viral marker might be useful for improving the quality of the histological or cytological evaluation of cervical specimens. Enhanced expression of a cyclin dependent kinase inhibitor $\mathrm{p} 16^{\mathrm{ink} 4 \mathrm{a}}$ can be used to increase the specificity and interobserver agreement of the histological assessment of cervical lesions (Klaes et al, 188). In addition, it was shown that immunocytological staining for $\mathrm{p} 16^{\text {ink4a }}$ can identify dyskaryotic exfoliated cells in cervical smears (Friedrich et al, 129).

Few studies compared the quality of HPV detection systems, and there were contradictory results with respect to sensitivity and type specificity of the various systems (de Sanjosé et al, 220; Perrons et al, 302; Molijn et al, 254). These discrepancies between different HPV detection systems, which are in wide clinical use, show that there is an urgent need for standardisation and quality control - an indispensable prerequisite if HPV testing is to be integrated into clinical practice. This problem has been recognised and led to the establishment of the "first human papillomavirus international quality control proficiency panel" at 
this meeting, an activity which should be supported by the scientific community and by industry.

HPV TESTING AS A TRIAGE METHOD FOR CYTOLOGY RESULTS OF UNDETERMINED NATURE Several studies concerned the value of HPV testing for triaging women with a diagnosis of ASCUS or low grade CIN (Solomon et al, 181; Ronco et al, 224; McGoogan et al, 256; Ponce et al, 294). Ponce et al (294) reported an equal value of HPV testing (hybrid capture II (HC II)) and expert cytology in predicting HSIL in women with ASCUS/LSIL. Ronco et al (244) showed that the use of HPV detection to triage women with cytological evidence of ASCUS and LSIL could reduce substantially the number of colposcopies, with little loss in histological detection of CIN2/3. Solomon et al (181), reporting from the ALTS trial (a randomised, multicentre trial comparing three management strategies for women with cytological diagnoses of ASCUS or LSILimmediate colposcopy, conservative management, or colposcopic triage using HPV DNA testing added to repeat thin layer cytology), showed that the negative predictive value of a negative HPV DNA test was high in all groups of study subjects, but that the positive predictive value of finding HPV was greatest in older women (>30) with ASCUS as a referral diagnosis on cytology. Thus, HPV testing was clearly superior to cytology at the expense of a referral rate of $56 \%$ for colposcopy. In another study including 270 women with a borderline or mildly dyskaryotic smear, no cases of HSIL would have been missed by HPV testing (McGoogan et al, 256). The authors conclude that HPV testing is likely to be useful in triaging women with low grade cervical abnormalities.

The value of HPV testing in the triaging of women with ASCUS seemed to be consistently superior to repeated cytology for the detection of HSIL. HPV testing in the triaging of LSIL cases is less consistent because of the high prevalence rates of HPV DNA found in some studies, such as ALTS. However, many authors noted the difficulties of comparing studies because of the additional variability introduced by the cytology methods used (standard $v$ expert $v$ liquid based) and the use of different axes of classification of the neoplastic lesions (CIN, the Bethesda system (TBS), Pap, and others).

HPV TESTING AS A PRIMARY SCREENING METHOD The validity of HPV testing in screening programmes was investigated in various cohorts (Franco et al, 180; Meijer et al, 182; Kulasingam et al, 183; Schneider et al, 184; Iftner et al, 185). In the Newfoundland study (180), the adjusted sensitivity for cytology for detection of HSIL with cut point LSIL was $28 \%$, and for HPV testing $68 \%$, at the expense of specificity, which was $90.6 \%$ for HPV testing versus $99.1 \%$ for cytology. A combination of HPV testing and cytology yielded a negative predictive value of $100 \%$ for the detection of high grade CIN. HPV screening in addition to cytology increased efficacy significantly.

In the Dutch screening programme (182), the prevalence of HPV DNA in cytological negatives was $3.4 \%$. Cytology negativity and high risk HPV positivity was associated with HPV persistence in $60 \%$ and led to abnormal cytology in $26 \%$ of HPV positives. Cytology positivity and high risk HPV positivity was associated with HPV persistence in $70 \%$ and led to high grade CIN in $24 \%$.

In the Seattle cohort (183), Thin-Prep cytology showed a sensitivity of $56 \%$ for the detection of CIN2/3, compared with a sensitivity of $67 \%$ by PCR based HPV DNA testing and $68 \%$ for HC II. Specificity for cytology was 93\% and for PCR 91\%, compared with $79 \%$ for HC II. With cytology, $12.5 \%$ of women had to be referred for colposcopy. Comparable figures were $15 \%$ using PCR and $23 \%$ with HC II. Thus, PCR had a higher sensitivity and comparable specificity when compared with liquid based cytology.

Two German studies compared the performance of routine cytology and HPV testing by GP5+/6+ PCR (Schneider et al, 184) or HC II (Iftner et al, 185). The sensitivity of cytology for the detection of HSIL was $20 \%$ (184) and $34 \%$ (185), respectively. HPV testing showed a sensitivity of $89 \%$ and a specificity of $94 \%$ in both studies. The positive and negative predictive values for HPV testing using PCR were $35.8 \%$ and $99.6 \%$, respectively.

SELF SAMPLING FOR HPV TESTING: A POTENTIALLY IMPORTANT DEVELOPMENT Several studies reported the value of self collected samples for HPV testing by PCR systems (Harper et al, 186; Nobbenhuis et al, 265; Mire et al, 275) or HC II (Salmerón et al, 270; Hillemanns et al, 303) compared with physician directed sampling. These studies concluded that HPV detection rates in self collected vaginal samples were similar to those in physician collected cervical samples, and that the use of self collected samples might be useful in cervical cancer screening in underserved women and women in developing countries (303 and 265). In a similar vein, Brinkman et al (274) showed that HPV detection rates using a PCR based urine test agreed reasonably with those derived from matching cervical swabs, raising the possibility that such samples might be useful.

ACCEPTANCE OF HPV TESTING BY THE POPULATION AND THE MEDICAL COMMUNITY An important prerequisite for the acceptance of HPV testing by individuals undergoing screening is the evaluation of socioeconomic and psychological factors, counselling of affected individuals, and information to the public, issues that were considered in various studies (Johnson et al, 228; Phillips et al, 229; Whynes et al, 230; Flores et al, 296). The clinical workshop included special presentations on the topics of "Counseling persons with HPV infections" (Steben, 008) and "Medical, social and ethical issues and challenges of HPV infections: what can we do?" (Alexander, 007). 
HPV IN IMMUNOCOMPROMISED INDIVIDUALS

The natural history of HPV might have to be redefined based upon the information gathered in immunosuppressed individuals (Hoesley et al, 154; Jin et al, 197): $76 \%$ of HIV positive women were found to be HPV positive by nested PCR, with 65\% harbouring multiple infections and with one third of isolates belonging to the phylogenetic subgroup A3. It is concluded that HPV infection might be acquired early in life, and that it might be part of the commensal viral microflora of the skin and the anogenital mucosa, with sporadic subclinical shedding caused by transient reactivation in specific settings such as immunosuppression and overt disease being unusual.

HPV ECONOMICS

HPV 2000 introduced the topic of HPV economics to stress the relevance of conducting cost-benefit analyses and studies evaluating quality of life. Most of the work presented did so in relation to screening and treatment of preinvasive neoplastic lesions through conventional cytology screening programmes or through primary HPV testing.

The addition of HPV testing was favourably evaluated as an adjuvant to the follow up of HIV positive women (Goldie et al, 267), and as a single lifetime screening method in low resource settings (Goldie et al, 693; SherlawJohnson et al, 260; Kulasingam et al, 272). HPV testing when combined with a low technology treatment, such as cryosurgery for HPV positive women, resulted in cost saving (Goldie et al, 693). An ongoing project in Vietnam showed that five yearly cytology based screening programmes were both feasible and cost beneficial (Chan Hung et al, 694). Quality of life issues about HPV testing (self sampling acceptability, cost acceptability, diagnostic related stress) form a novel research area that presents several challenges before primary HPV testing can be implemented (Flores et al, 296; Johnson et al, 228; Philips et al, 229). Some exercises based on mathematical models for predicting outcomes of screening programmes under a variety of assumptions were also presented (Gallivan et al, 259; Whynes et al, 230) and may become an area of increasing interest.

\section{Treatment of HPV infections and of HPV} related cancer

In vitro studies: various gene therapeutic modalities were defined such as HSV thymidine kinase (HSV-TK) (Sethi et al. 326), HSV vectors (Kari et al, 345), HPV-16 E2 derivates (Hoffmann et al, 333), and anti-HPV hairpin ribozymes (Enriquez-Aragon et al, 328). In addition, growth factor inhibition (Michael et $a l, 342$ ) and endothelin receptor antagonists (Venuti et al, 313) were used. The in vitro results of these studies look promising and development to clinical application is a realistic goal.

In vivo studies in animals: for preclinical studies, HPV-6 or HPV-11 induced human xenografts were used in the severe combined immunodeficient (SCID) mouse model (Bonnez et al, 339; 340; 341). It was shown that the antisense oligonucleotides ORI-1001 complementary to the 5' end of HPV-6 E1 mRNA has an inhibitory effect on the growth of HPV-6 induced human condylomata (339).

In vivo studies in humans: experiences with indole-3-carbinol (I3C), a compound found in cruciferous vegetables that detoxifies carcinogens and alters oestrogen metabolism, were reported by Bell et al (311) and Da-Zhi Chen et al (312). It was shown that I3C and its metabolite DIM caused apoptotic changes in cervical cancer cells in vitro. In vivo, HPV transgenic mice exposed to oestrogen and fed I3C showed evidence of apoptotic cells in their cervical epithelium (312).

To evaluate the effectiveness of I3C in humans, 27 patients with biopsy confirmed CIN2 or 3 were randomised to oral I3C or placebo (311). Eight of 17 patients treated for 12 weeks had complete regression of lesions. None of the 10 patients in the placebo group experienced regression. In another study, a fusion protein consisting of the heat shock protein Hsp65 and Mycobacterium bovis BCG linked to the HPV-16 E7 protein was constructed. In a randomised double blind placebo controlled trial, three monthly subcutaneous doses were applied to 10 patients with high grade anal intraepithelial neoplasia (Goldstone et al, 347a). All patients had a clinical response after a course of three doses.

Immunomodulatory substances such as imiquimod (Ikenberg et al, 310), leukonorm (Metzner et al, 322), viscum album (Romero Montes et al, 327), and a combination of iscador and interferon (IFN) (Basta et al, 318) were used in various studies for the treatment of CIN and anal condylomata in phase 2 trials and showed promising responses with tolerable side effects. Medical treatment of LSIL emerged as an area of growing research interest and a plausible alternative to reduce surgical overtreatment.

HPV DNA AS A PROGNOSTIC FACTOR IN CIN CASES AFTER TREATMENT

Studies evaluating the presence of HPV DNA after surgical treatment of CIN (Kjellberg et al, 196; Tachezy et al, 242; Nobbenhuis et al, 317; Ramírez Porras et al, 320; Simon et al, 323; Bettinger et al, 193; Elfgren et al, 329) showed that the detection of HPV increases the risk of recurrent disease. HPV persistence is rare after conisation with free margins, but not after conisation with positive margins (323). A high risk HPV positive test three months after treatment is more predictive for residual or recurrent CIN disease than abnormal cytology (317). Persistence of HPV infection after treatment was more common in patients less than 35 years of age and with a history of cryotherapy (329). Conisation resulted in a decline of cervical HPV IgA antibodies whereas cryotherapy had much less effect (329). Using HPV-16 E6 and E7 peptides, a cellular immune response was seen in disease free patients but not in those with disease recurrence (Sarkar et al, 314). 
HPV TESTS AS PROGNOSTIC MARKERS IN INVASIVE CANCER

In patients with invasive cancer (Lizano et al, 325), viral DNA sequences were detected in the plasma of four of five patients with recurrence and all three patients with tumour persistence, but in only one patient of 17 with regression. A prognostic effect of the presence of HPV antibodies, HPV DNA (Bravo et al, 338), certain HPV types (Lo Keith et al, 332; Dreier et al, 335), VEGF serum concentrations (Bachtiary et al, 336), or marker chromosomes (Macville et al, 344) was shown in case series, but larger prospective series are needed to substantiate these preliminary observations.

\section{Immunology and vaccines}

Recognition of the association between HPV infection and cervical cancer in the 1980s fuelled interest in HPV immunology, and in the development of vaccines to prevent infection. However, data were initially slow to emerge, in part because two problems hindered research, namely: (1) PV could not be grown in the laboratory as a source of antigen for the development of serological tests and for conventional killed vaccines; and (2) no PV could be identified that infected an immunologically well characterised laboratory animal.

Improved systems for expressing genes in eukaryotic cells through the 1990s has allowed a partial solution to the first problem, which is exemplified by the production of VLPs and the recent resolution of a crystal structure for the capsid of HPV-16 (Chen et al, 359), and has led to a considerable worldwide interest in the development of vaccines to prevent and to treat PV infection. In addition, epithelial raft cultures (Thomas et al, 535) have recently allowed the production of authentic virions from epithelial cells infected in vitro. The lack of an animal model for the study of PV immunology has been circumvented by the development of more robust tests for cell mediated immunity to defined antigens in humans, including tetramer and ELISPOT technology, and key experiments on the immunological consequences of PV infection can now be undertaken in the natural host. Such research has demonstrated the ability of PV to elicit both humoral and cellular immunity and, possibly as a consequence of preferential uptake by dendritic cells (DCs) into the major histompatibility complex (MHC) class I presentation pathway, PV VLPs can also induce host protective cytotoxic $\mathrm{T}$ cell responses. Interest in PV immunology has now extended outside the narrow confines of viral epidemiology, and abstracts on PV immunology and immunoserology, and on vaccine development, accounted for nearly a quarter of the material presented at the workshop.

\section{SEROLOGICAL RESPONSES TO HPV INFECTION}

Data presented from prospective studies of infection with genital genotypes of HPV suggest that serological responses to the viral capsid are slow to appear, when compared with most viruses. Only $50 \%$ of individuals acquiring HPV DNA of genotype HPV-16 develop capsid specific antibody within four years of infection, with a median time to seroconversion of over six months (Carter et al, 057). HPV-18 seroconversion is similarly slow, whereas seroconversion for HPV-6b is apparently faster, and occurs at the same time as the acquisition of viral DNA (057). The definition of a positive serological reaction to the capsid remains mathematical (Stone et al, 056) because the identification of a cohort of individuals never infected with a particular HPV genotype is not straightforward, and there is also some evidence of genotype crossreactivity for serological reactions (Combita et al, 412). Hence, the usefulness of serology for epidemiological studies is limited. For virus encoded nonstructural proteins, serological tests are not yet as well standardised as for PV virions. Antibody to the E7 protein, which is expressed in acute infection (Hamsíková et al, 397) and also in many PV associated tumours, is seen in about $50 \%$ of subjects with invasive cervical cancer. However, it is not seen after acute HPV infection with several genotypes (Gaenzler et al, 398), even in the presence of antibody to the L1 capsid protein. Low amounts of antibody reactive with peptides derived from other open reading frames (E2 and $\mathrm{E} 4$ ) are seen in some patients with HPV infection and invasive cancer (Sehr et al, 399), although sensitivity and specificity for infection appear to be low. These results are reminiscent of the results obtained with L1 protein before the use of correctly conformed VLP substrate.

CELL MEDIATED IMMUNE RESPONSES

Several presentations describing the consequences of HIV infection on the natural history of HPV infection confirmed that HPV infection is more likely to persist and less likely to respond to treatment in individuals with systemic immune suppression (Palefsky, 349; Ebrahim et al, 350). These data strongly suggest a role for cell mediated immune responses in the control of HPV infection, as would be expected from data for other viruses. The newer assays (such as ELISPOT and tetramer technologies) allow more precise measurement of virus specific cytotoxic $T$ cells (CTLs) and to some extent of virus specific $T$ helper responses (Steele et al, 384). Assay reproducibility and sensitivity remain important issues, and there is only a limited number of defined viral protein epitopes restricted by defined MHC class I and II haplotypes. T cell assays, to be suitable for immunoepidemiology, or for modelling mechanisms of disease control, need to: (1) produce positive results that are reproducible from day to day in the same patient, (2) correlate with the genotype of clinical HPV infection, and (3) demonstrate MHC restriction and antigen specificity. Few of the presentations gave results from human studies that met these stringent criteria. Positive control reagents, including immortalised antigen specific $\mathrm{T}$ cell lines, using telomerase reverse transcriptase (TERT) transfer (Hooijberg et al, 427), promise to help with assay standardisation in the future. 
Cytotoxic $\mathrm{T}$ cell mediated immune responses are not easily demonstrable after natural HPV infection. Low frequencies of CTLs specific for the E6 protein of HPV-16 were associated in one study with the resolution of cervical infection, whereas E7 specific CTLs were found both in patients with regression and in patients with persisting infection (Nakagawa et al, 354). Of potential importance for vaccine development, some E7 specific CTL epitopes were shown to be crossreactive between HPV-16 and HPV-52 (Youde et al, 357). More studies in this area will be needed to determine whether host protective crossreactive responses exist after natural HPV infection or vaccination. Virus specific T helper cells are, predictably, harder to demonstrate than specific CTL responses, given the much lower frequency of $\mathrm{T}$ helper cells after viral infection. The E7 protein contains sequences recognised as MHC class II restricted epitopes by most normal subjects (van der Burg et al, 358), as does E2 (de Jong et al, 383; Dillon et al, 408). Patients with cervical cancer but not patients with squamous intraepithelial lesions (SIL) respond to E6 peptides in vitro (Timmins et al, 353), and patients with progressive cervical disease respond less to E7 peptides (Luxton et al, 400), although multiple in vitro restimulations are required to elicit positive proliferative responses. Responses to E6 and E7 peptides are better in patients who are disease free after treatment (Sarkar et al, 314). Cytokine secretion by $\mathrm{T}$ cells in response to E6 and E7 proteins is altered in recurrent respiratory papillomatosis (RRP) (DeVoti et al, 406), suggesting that failure of resolution after infection might reflect immune deviation to a more $\mathrm{T}$ helper type 2-like immune response. In vivo testing of delayed type hypersensitivity (DTH) to antigen is a cheap, simple, and robust technique for measuring $\mathrm{CD} 4^{+} \mathrm{T}$ cell responses to viral antigens worthy of further investigation, and in one study DTH to E7 peptides was found to be more common in regressing than progressing CIN (Höpfl et al, 351), in general support of the hypothesis that cell mediated immunity is important for the regression of HPV infection. In keeping with this, regressing as opposed to persistent CIN appears to be associated with increased numbers of activated $\mathrm{T}$ cells locally within the lesion (Petry et al, 352). Local immune responses to PV antigen in the female genital tract most probably reflect systemic as well as mucosal immunity and, perhaps in consequence, $\operatorname{Ig} \mathrm{A}$ anticapsid antibody in the genital secretions, although common in HPV infection, is not a marker for persisting local infection (Pretet et al, 396).

PV EVASION IN HOST IMMUNITY

The lack of demonstrable immunity to nonstructural PV proteins after acute HPV infection, together with the slow and variable response to the capsid proteins, suggests that there is a problem with the generation of PV specific immunity. In contrast, PV VLPs are highly immunogenic when delivered as a vaccine without adjuvant, stimulate DCs (Rudolf et al, 355; Lenz et al, 395), and induce immune responses including CTLs. This paradox can be explained by noting that PV infection is non-lytic and superficial, and because most virus genes are expressed at low levels they might simply be ignored by the immune system. This has been demonstrated in a skin transplant model using HPV-16 E7 transgenic skin, where rejection only occurs after the administration of powerful proinflammatory signals, and is mediated via MHC class II dependent mechanisms (Frazer et al, 378). Alternatively, if PV antigen expression is increased, presentation in skin without inflammation may be tolerogenic, because skin associated E7 antigen can be cross presented by bone marrow derived DCs and anergise specific T cells, at least in the mouse (Doan et al, 356). In addition, and in common with most viruses, PV genes encode mechanisms for modulating both the induction of the host immune response and virus specific effector mechanisms. Initiation of the innate and adaptive immune responses to PV antigens may be impaired by E7 mediated downregulation of the host response to IFN (Barnard and McMillan, 579; Nees et al, 630). E5 and E7 also interfere with the TAP (transporter associated with antigen presentation) antigen transporter mechanism in infected cells (Vambutas et al, 360), and with MHC class I presentation of antigen. Local proinflammatory chemokines (Fife et al, 388) and cytokine B (Bermúdez Morales et al, 379; Jacobs et al, 416) production is altered in PV infected as opposed to uninfected keratinocytes. Additional mutations in the antigen processing and presentation pathways are seen in cervical cancers (Garrido, 370): SCID/human (HU) tumour grafts, facilitated with steroid, might prove an interesting model to examine defects of the antigen presenting machinery in tumours, free of stroma (Baines et al, 625).

PROPHYLACTIC VACCINES AGAINST PV: PHASE I AND II HUMAN TRIALS

All existing effective prophylactic vaccines against viral infection induce neutralising antibody, and none is thought to produce sterile immunity. Some epidemiological evidence was presented suggesting that the development of an antibody response in humans protects against repeat PV infection (Ho et al, 058), which is reassuring for the development of prophylactic vaccines, because to date the data supporting such vaccines have been based on studies in animal models. Most work on prophylactic vaccine development has focused on vaccines that are based on VLPs comprising the PV L1 capsid protein, produced in yeast or insect cells (Schiller, 348). Were such vaccines to be available, one study indicated that they would be generally acceptable to high risk communities (Lazcano et al, 446). Early phase clinical trials of vaccines for HPV-11 (Fife et al, 364) and HPV-16 (Harro et al, 362; Poland et al, 363; Balsley et al, 366) were presented by several groups. HPV-11 VLPs produced neutralising antibody for up to 18 months. HPV-16 VLPs are similarly immunogenic even without 
adjuvant. In general, antibodies are PV genotype specific, and immunogenic at relatively low antigen doses, although some vaccines might have stability problems. However, in the beagle dog, VLP delivered intranasally, although inducing a good mucosal IgA response, failed to protect against virus challenge (Yuan et al, 369), suggesting that systemic vaccine delivery may have advantages even for mucosal PV genotypes. Although no polynucleotide vaccines have been tried in humans, DNA encoding canine oral papillomavirus (COPV) capsid proteins protects dogs against viral challenge (Stanley et al, 375) and may clear virus from existing infections (Moore et al, 452): modification of codon usage of the PV capsid genes to match that of more conventional mammalian genes improves the immunogenicity of PV DNA vaccines in animals (Liu et al, 451), and may therefore facilitate their use in humans.

Minor HPV variants share epitopes (Pastrana et al, 368), but crossreactivity of neutralising antibody between major types is limited, although the requirement for multicomponent vaccines can be overcome with chimaeric VLPs displaying two or more types of HPV, at least in animals (Wilson et al, 462). PV neutralisation assays, which until recently have relied on a labourious in vivo xenograft model, are now available for in vitro use, based on reporter genes (Yeager et al, 371) or detection of viral E2 mRNA by reverse transcription PCR (Hall et al, 426). Such assays should allow the validation of vaccines for the less common genotypes based on surrogate markers of efficacy, without the need for extensive clinical trials.

Several studies (Chackerian et al, 389; Liu et al, 390) examined the use of PV VLPs as delivery systems for other antigens. VLPs are potent immunogens that can induce DTH in humans (Lian et al, 431), and $\mathrm{T}$ cell proliferation and occasional CTL responses in chimps (Pretet et al, 396). Chimaeric VLPs can activate DCs (Balmelli et al, 391; Lenz et al, 395) and induce epitope specific CTL responses with ex vivo human cells, and in vivo in mice. Adjuvants such as CpG DNA enhance VLP induced mucosal immunity (Gerber et al, 465), although as mentioned above a role for mucosal immunity in the control of PV infection remains to be demonstrated. Although most VLPs for vaccine use have been produced in yeast or insect cells, alternative expression systems that might better suit developing countries are being explored, including bacterial systems (Escherichia coli, salmonella, BCG), which produce immunogenic and host protective L1 pentamers, and plant derived VLPs are also under development (Accardi et al, 456). Although PV capsid based vaccines are currently favoured for prophylaxis against PV infection, vaccines based on multiple nonstructural proteins were found in one study to protect against infection in the rabbit (Han et al, 374), an observation worth following up for combined prophylactic/therapeutic vaccines.
THERAPEUTIC VACCINES

Currently, there are no licensed therapeutic vaccines for humans, and no clear evidence of which PV viral protein(s) might produce a therapeutic immune response to PV infection. Cottontail rabbits undergo regression of cottontail rabbit PV (CRPV) induced warty lesions after the administration of a polynucleotide vaccine encoding E1, E2, E6, and E7 (374), and the same proteins fused to ubiquitin are equally successful (Leachman et al, 455). Although data on efficacy in natural PV infection are limited, a wide array of potential vaccine delivery systems have been tested for immunogenicity in transplantable tumour models. Immunotherapy with polynucleotide vaccines, either unmodified (Velders et al, 382; Doan et al, 441; Smahel et al, 445; De Marco et al, 449), codon modified (Liu et al, 451), shuffled to avoid potentially harmful consequences of gene expression (Osen et al, 372), or fused with ubiquitin to improve antigen presentation (Brandsma et al, 373), has been effective in the prevention or elimination of PV protein expressing transplantable tumours. Similarly, vaccines based on cell derived (Navabi et al, 447) or recombinant (Giannouli et al, 429; O'Neill et al, 438) proteins, derivative peptides (Melief et al, 437), recombinant bacteria (Benyacoub et al, 450; Revaz-Fellay et al, 453; Maclean et al, 457), recombinant viruses (Jifeng et al, 430; Chen et al, 436; Herd et al, 440), and chimaeric virus-like particles (Liu et al, 386; Street et al, 439; Liu et al, 442; Shi et al, 448; Revaz-Fellay, 453; Kaufmann et al, 460) are all protective in at least one animal tumour model system. Responses to such vaccines can be improved by cytokines (Hung et al, 434), by targeted delivery systems (Wang et al, 435; Michel et al, 458), and by new adjuvants such as Hsp (Kadish et al, 365; Randall Chu et al, 377; Cheng et al, 433), whereas "mix and match" prime boost strategies (Chen et al, 381) produced improved responses in animal models when compared with single delivery systems. The human trials of these therapeutic vaccines to date have been more limited. They have shown safety and some immunogenicity of $\mathrm{E} 7$ based vaccines. A fusion of Hsp with E7 induced transient proliferative responses to E7 peptides in $\mathrm{T}$ cells in four of the eight subjects immunised. E7 peptides induce some immune responses but CIN regression was no more than might be expected without vaccine (Kast et al, 367), and no regression of cervical cancer was reported with any vaccine (Khleif et al, 380), although many trials are ongoing. Ex vivo DCs pulsed with E7 induce E7 specific CTLs (Evans et al, 410; Nonn et al, 415; Hermonat et $a l, 464)$, but efficacy data in humans are not available.

It will not be feasible to conduct human efficacy studies of all the available PV therapeutic delivery systems and antigens, and to date no single immunological assay has shown a correlation with efficacy of immunotherapy for PV induced disease in humans or in natural animal PV infection. How can we progress from here? The usefulness of further studies of vaccine efficacy using transplantable E7 expressing 
tumours in the mouse is limited, and although the cottontail rabbit and the beagle dog provide attractive models for testing immunotherapy, the natural history of PV infection in these animals does not closely resemble that of HPV infection in humans. Tumour xenografts to SCID/HU and transgenic skin/tumour models might more closely resemble natural human PV infection, but are not ideal for establishing vaccine efficacy because the immune system of these animal models is compromised. It will probably be necessary to validate surrogate markers of vaccine therapeutic efficacy in phase I clinical trials, and discard non-valid markers. Phase I trials of immunotherapy for warts/anal intraepithelial neoplasia (AIN)/ $\mathrm{CIN} /$ vulvar intraepithelial neoplasia (VIN)/ RRP will be preferable for evaluating surrogate efficacy markers against outcome because problems with mutated antigen presentation machinery, and with the effects of previous treatments, complicate interpretation of the data in patients with cancer. True validation of the surrogate efficacy markers will not be possible from such studies unless studies are blinded, placebo controlled, and randomised because of the subjective nature of the outcomes from such studies.

\section{Biology of PV and virus and host interactions}

Contributions to biology improved the basic knowledge of this virus family and paved the way for epidemiology, diagnosis, and immunology by identifying and cloning HPV types, demonstrating their association with benign and malignant tumours in humans, and revealing molecular mechanisms of immortalisation and tumour progression. This provided reagents and tools for epidemiology and HPV specific, diagnostic screening programmes. It also defined targets for prophylactic and therapeutic vaccination.

\section{VIRAL ONCOPROTEINS}

Relevant to oncogenicity are the viral proteins E6, E7, and to a lesser degree E5. They are mitogenic and modulate apoptosis; in addition, E6 and E7 induce genetic instability. A prime cause for chromosomal instability is E6 mediated degradation of the cellular protein p 53 by the ubiquitin-proteasome pathway. Degenkolbe et al (639) showed that E6 targets p53 in yet another way by binding to the transcriptional coactivator $\mathrm{CBP} / \mathrm{p} 300$ required by $\mathrm{p} 53$, thereby inactivating this factor. It is interesting to note that E6 of bovine PV-1, which does not induce the degradation of $\mathrm{p} 53$, can also downregulate $\mathrm{CBP} / \mathrm{p} 300$, and that this interaction appears necessary but not sufficient for cell transformation. Regarding the interaction of $\mathrm{E} 7$ with the retinoblastoma protein $\mathrm{pRb}$, the importance of enhanced $\mathrm{pRb}$ degradation in comparison with the molar E7-pRb interaction has been stressed (Gonzalez and Münger, 587). Phosphorylation of the E7 protein has been shown to be crucial for promoting $S$ phase entry (Chien et al, 588). Phosphorylation is inhibited by a macrophage inhibitory factor related protein complex, which is no longer expressed in HPV transformed cell lines and in high grade squamous intraepithelial lesions, indicating that loss of this complex and increased phosphorylation of E7 might contribute to malignant transformation (Tugizov et al, 589).

Regarding genetic instability of persistently infected keratinocytes, it is of interest that E7 uncouples centrosome duplication from the cell division cycle and that E6 allows the accumulation of centrosome abnormalities (Duensing and Münger, 471). This will increase the likelihood of chromosomal mis-segregation followed by genomic changes.

In addition to its role in the epidermal growth factor receptor signal transduction pathway, HPV-16 E5 has been shown to downregulate the cyclin dependent kinase inhibitor p27 (Pedroza-Saavedra et al, 583).

All oncoproteins are involved in the protection of the infected keratinocyte from the host immune response. HPV-16 E6 leads to reduced expression of E-cadherin, which has been postulated to play a role in the retention and localisation of Langerhans cells (Matthews et al, 665). The E7 protein blocks the antiviral and antigrowth activities of IFN- $\gamma$, possibly by binding to cellular p48 (Barnard and McMillan, 579). However, it does not alter the activity of IFN- $\alpha$. In cells transformed by E5 or the homologous E8 protein of bovine PV-4, the export of MHC class I molecules to the cell surface is downregulated, impairing antigen presentation, and thereby helping the virus to escape immune recognition (Ashrafi and Campo, 592).

\section{REPLICATION AND TRANSCRIPTION}

The viral proteins E1 and E2 are the major players in the control of replication and transcription. E7 seems to promote DNA synthesis by directly activating the cyclin dependent kinase CDK2 and enhancing the phosphorylation of histones and possibly E1 and E2 (He and Fisher, 586). The interaction of E1 and $\mathrm{E} 2$ with $\mathrm{CBP} / \mathrm{p} 300$ has been shown to stimulate transcription and replication in HPV-18 and HPV-8 (Lee and Choe, 526; Müller et al, 540). Furthermore, E2 interacts with the transcription factor NF-IL6 to synergise in transcriptional activation (Hadaschik et $a l, 541)$. A new mechanism for the repression of transcription by E2 has been proposed. The E8-E2C fusion protein of HPV-31 not only represses by competition for DNA binding with transactivation competent full length E2, but also possesses an active repression domain in the E8 part, which mediates repression over long distances (Zobel et al, 531).

The very low concentrations of E1 in PV infected cells always suggested that this replication initiator is tightly regulated. It has now been shown that E1 is degraded by the ubiquitin-proteasome pathway unless protected in a complex with cyclin E-CDK2 before the start of DNA synthesis (Malcles et $a l, 622)$. E1 independent replication has been described for HPV types 16, 18, and 31, starting from origins outside the long control region, and is possibly functional during the 
non-productive state of the viral life cycle or during latency (Kim and Lambert, 528). This is important news regarding antiviral therapeutic approaches targeting the E1 protein.

The E1-E4 fusion protein has so far been regarded mainly as a factor in virus maturation but now also seems to play a role in replication. It interacts with the human replication initiation protein $\mathrm{Mcm} 7$ and thereby inhibits cellular DNA synthesis (Roberts et al, 631). This should allow the recruitment of host cell factors to sites of viral DNA synthesis.

New ways to control transcription and replication have been revealed by the analysis of nucleosomes specifically positioned on the viral enhancer and the E6 promoter, and flanking nuclear matrix attachment regions, which bind the differentiation regulated CCAAT displacement protein (CDD) factor (Bernard et al, 532). In transient transfection assays, these act as silencers of transcription by deacetylating the nucleosomes. However, after integration of the viral DNA, the matrix attachment regions change into potent enhancers, which may partially explain the deregulation of viral gene expression seen after the integration of viral genomes into cellular DNA in the course of tumour progression.

INTRATYPE VARIANTS

A newly identified variant of the CRPV differs in biological behaviour from the prototype. $\mathrm{CRPVb}$ induced papillomas regress in the vicinity of still proliferating CRPVa induced papillomas (Nasseri et al, 674; Nonnenmacher et al, 479). Differences in risk for persistence and malignant conversion were mapped to the control and E6-E7 regions of the variant genomes (differences in wart regression especially to E6) and were strongly dependent on the DR-DQ haplotype of the host.

Similar important differences in oncogenic potential seem to exist among variants of HPVs. The oncogene promoter of so called Asian-American and North American variants of HPV-16 turned out to be about three times more active than the European prototype (Kämmer et al, 548), and African 1 isolates from penile cancers were more active than the respective prototype (Tornesello et al, 518).

Certain E7 variants of HPV-45 have increased transforming potential (Geisbill et al, 515). The association of HPV-16 E6 variants with cervical cancer appear to be linked to human MHC (HLA) class II distribution (Voglino et al, 650), which may indicate that the risk of specific E6 polymorphism is linked to host immune surveillance, analogous to the above data on CRPV.

\section{LIFE CYCLE}

Adsorption of the virus to its host cell is the first step in the life cycle. So far, $\alpha 6 / \beta 4$ integrin has been put forward as a potential receptor but turned out to be not obligatory for bovine PV-4. Studies with HPV-11 VLPs pointed to glycosaminoglycans as receptors. A convincing study with HPV-33 pseudovirions showed that heparan sulphate proteoglycans are crucial for HPV binding because heparinase completely prevented infection (Giroglou et al, 619). The $\mathrm{N}$-terminal region of $\mathrm{L} 2$ has been shown to bind to cells and to enter the cytoplasm (Kawana et al, 621). This suggests that the minor capsid protein plays an important role in virus-host cell interaction.

Organotypic raft cultures of keratinocytes were introduced long ago into PV research but it is only now possible to produce infectious viral stocks with titres of 1000 and more in vitro (Meyers et al, 632). This will be useful in future studies of the viral life cycle.

\section{RELEVANCE OF CELLULAR GENES}

The importance of cellular genes in tumour progression has been undisputed. Using the array technique, several groups have started to collect data on differential gene expression, at the moment mainly using archival material. Other approaches are already more focused. The E6 mediated induction of transcription of human TERT (hTERT), the catalytic subunit of the telomerase, is known to be an important step in immortalisation. This is followed by the loss of cellular telomerase inhibitors. Using microcell mediated chromosomal transfer, Snijders et al (485) and Backsch et al (486) demonstrated telomerase suppression by chromosomes 3, 4, and 6, thereby mapping repressors, the loss of which is involved in HPV mediated immortalisation. Another putative senescence gene locus has been mapped to the short arm of chromosome 10 (Poignée et al, 476).

A great success has been achieved in characterising the genetic abnormality of patients with $\mathrm{EV}$, who are susceptible to infection with the subgroup of HPV associated with this disease. Genetic linkage analyses on EV consanguineous families has recently identified a first susceptibility locus on chromosome 17q25 and a second locus has now been mapped to chromosome $2 \mathrm{p} 21-24$ (Ramoz et al, 628). This is the first identification of genomic regions involved in the control of HPV infections. It is of particular interest that the EV susceptibility locus on chromosome 17 overlaps with a psoriasis susceptibility locus, in view of recently published papers on the high prevalence of EV associated PVs in skin lesions of patients with psoriasis.

\section{Summary and prospects}

CLINICAL

In terms of prospects for the near future, it seems that the most exciting developments will relate to the addition of HPV testing to cervical cancer screening programmes. In this regard, the Dutch trial, which ultimately will involve 44000 women (Meijer et al, 182), will yield important data in the next few years. Also, several cost-benefit analyses of HPV detection (Sherlaw-Johnson et al, 260; Goldie et al, 267; Kulasingam et al, 183) for screening for cervical neoplasia were presented at the meeting, and analyses of this type will be important in evaluating the feasibility of this approach to screening. The other important development in relation to screening is the potential applicability of HPV testing, performed perhaps on 
self collected samples, to screening for cervical neoplasia in underserved women and women in developing countries. Relatively little work has been done in this area, although there now appears to be a steady growth of activity. Finally, one other area about which we can expect to learn more in the near future is serological testing for HPV. New HPV detection systems have been created that should be used to improve the performance of currently used tests, especially with respect to HPV type specificity. Clinically useful prognostic markers and non-invasive treatments still have to be developed.

IMMUNOLOGY AND VACCINES

Improved assays to measure immune responses to PV antigens in humans will allow seroepidemiological studies to explore the natural history of PV infection, and the role of specific PVs in diseases in which the link with PV is not yet clarified. Studies of the immune responses associated with the resolution of PV infections in humans will pave the way for immunotherapy for PV associated diseases. VLP based vaccines are likely to play a part in preventing $\mathrm{PV}$ infection and hence cervical cancer in the near future. Studies of immunotherapy for PV infection will assist in evaluating the role of antigen specific immunotherapy in the control of PV associated and other cancers. PV VLPs have a promising future as candidate delivery systems for other antigens, both for prophylaxis and for treatment.

BIOLOGY

Several excellent studies were presented on novel functions of viral proteins, transcription control mechanisms, and intratype variants, which differ considerably in biological activities. The availability of pseudovirions and the use of raft cultures continued to be useful for unravelling the life cycle of PVs, which will allow new approaches for therapeutic interventions. Studies of virus-host interactions are beginning to profit from data from the human genome project and techniques such as microcell mediated chromosomal transfer or microarray analyses. These techniques enhance the understanding of the regulation of senescence and predisposition to PV infection. The role of PVs in the aetiology of skin cancer came into focus by identifying new cutaneous HPV types and through preliminary experiments on the biochemical activities of cutaneous HPV oncoproteins.

The conference was credited for CME purposes by the European School of Oncology (ESO), the Federation of European Cancer Societies, the American Medical Association (AMA), and by the Spanish CME University System (Consell Coordinador de la Formacio Medica Continuada). The Union Internatinonale Contre le Cancer (UICC) acted as a scientific sponsor. The HPV 2000 conference was supported by public grants from the European Commission (QLFK2-1999-30059 and HPCF-1999-00208), the National Cancer Institute of the US (R13CA90191 to the University of South Carolina School of Medicine), the II Pla de Recerca de Catalunya 1997/2000 (2000 ARCS 00111), and the Spanish Ministry of Education and Culture (C099-0430). Support was also received from private sponsors with interest in HPV research and clinical uses. The major sponsors were $3 \mathrm{M}$ Pharmaceuticals, $3 \mathrm{M}$ Farmacéutica, Digene, Merck Vaccine Division, Smith Kline Beecham, Abbott Cientifica, Cytyc Corporation, and Roche Diagnostics (for full credit description see www.hpv2000.com). The organisers of the HPV 2000 conference acknowledge the support and contribution of the Institut Català d'Oncologia for handling the administration and the staff at the Servei d'Epidemiologia i administration and the staff at the Servei d'Epidemiologia i Registre del Cancer, SERC and the Seccion de Ginecologia Oncologica (ICGON), Hospital Clinic i Universitari de Barcelona. The organisers acknowledge with gratitude the support received from all sources and wish to express the continuous independence of the organising committee in establishing the programme, inviting the coauthors of this review, and editing

1 ALTS Group. Human papillomavirus testing for triage of women with cytologic evidence of low-grade squamous intraepithelial lesions: baseline data from a randomized trial. $\mathcal{F}$ Natl Cancer Inst 2000;92:397-402.

2 Cuzick J, Sasieni P, Davies P, et al. A systematic review of the role of human papillomavirus testing within a cervica screening program. Health Technol Assess 1999;3:1-196.

3 Gross GE, Barrasso R, Human papillomavirus infection. In: A clinical atlas. Berlin: Ullftein Hosby; 1997:1-432.

4 Harwood CA, McGregor JM, Proby CM, et al. Human papillomavirus and the development of non-melanoma skin cancer. F Clin Pathol 1999;52:249-53.

5 Holowaty P, Miller AB, Rohan T, et al. The natural history of dysplasia of the uterine cervix. F Natl Cancer Inst 1999;91:252-8

6 Kuhn L, Denny L, Pollack A, et al. Human papillomavirus DNA testing for cervical cancer screening in low-resource settings. F Natl Cancer Inst 2000;92:818-25.

7 Manos MM, Kinney WK, Hurley LB, et al. Identifying women with cervical neoplasia. Using human papillomavirus DNA testing for equivocal Papanicolaou results. $F A M A$ 1999;281:1605-10.

8 Meijer CJLM, Walboomers JMM. Cervical cytology after 2000: where to go? f Clin Pathol 2000;53:41-3.
20ijer Cul

9 Nobbenhuis MAE, Walboomers JMM, Helmerhorst TJM, et al. Relation of human papillomavirus status to cervical lesions and consequences for cervical-cancer screening: a prospective study. Lancet 1999;354:20-5.

10 Ramoz N, Taieb A, Rueda LA, et al. Evidence for a nonallelic heterogeneity of epidermodysplasia verruciformis with two susceptibility loci mapped to chromosome regions 2p21-p24 and 17q25. F Invest Dermatol 2000;114: $1148-53$.

11 Rozendaal L, Westerga J, van der Linden JC, et al. PCR based high risk HPV testing is superior to neural network based screening for predicting incident CIN III in women with normal cytology and borderline changes. $\mathcal{F}$ Clin Pathol 2000;53:606-11.

12 Salmon J, Nonnenmacher M, Caze S, et al. Variation in the nucleotide sequence of cottontail rabbit papillomavirus a and $b$ subtypes affects wart regression and malignant transand b subtypes affects wart regression and malignant transf Virol 2000;74:10766-77.

13 Schiffman M, Herrero R, Hildesheim A, et al. HPV DNA testing in cervical cancer screening. Results from women in a high-risk province of Costa Rica. $f A M A$ 2000;283:87-93.

14 Stubenrauch F, Hummel M, Iftner T, et al. The E8E2C protein, a negative regulator of viral transcription and replication, is required for extrachromosomal maintenance of human papillomavirus type 31 in keratinocytes. $\mathcal{F}$ Virol 2000;74:1178-86.

15 Tindle RW. Vaccines for human papillomavirus infection and anogenital disease. Texas: R.G. Landes Company (Medical Intelligence, Unit 14), 1999.

16 Vernon SD, Unger ER, Williams D. Comparison of human papillomavirus detection and typing by cycle sequencing, line blotting, and hybrid capture. F Clin Microbiol 2000;38: line blottir $651-5$.

17 Walboomers JMM, Jacobs MV, Manos MM, et al. Human papillomavirus is a necessary cause of invasive cervical canpapillomavirus is a necessary cause of inv
cer worldwide. $\mathcal{F}$ Pathol 1999;189:12-19.

18 Wells SI, Francis DA, Karpova AY, et al. Papillomavirus E2 induces senescence in HPV-positive cells via pRB- and p21(CIP)-dependent pathways. EMBO f 2000;19:576271

9 zur Hausen H, Papillomaviruses causing cancer: evasion from host-cell control in early events in carcinogenesis. $\mathcal{F}$ Natl Cancer Inst 2000;92:690-8. 